\title{
Dove vivono i sudafricani?
}

\section{Enzo Caffarelli}

PUBBLICATO: 08 OTTOBRE 2019

\section{Quesito:}

Numerosi lettori chiedono se sia più corretto chiamare Sudafrica o Sud Africa lo Stato che occupa la parte più meridionale del continente africano, ufficialmente Repubblica Sudafricana. Alcuni si domandano se le due varianti grafiche possano opportunamente individuare l'una lo Stato e l'altra il cono sud dell'Africa.

\section{Dove vivono i sudafricani?}

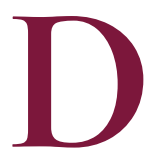

ue le piste da seguire: la tradizione nell'uso italiano di questi coronimi e la grafia nei corrispondenti in altre lingue. Andrà premesso che il Paese è indipendente (dal Regno Unito) dal 3I maggio I96I, ma che l'odierno Sudafrica fu unificato gia dal igo9 e che dal I9I4 venne formalmente costituito come dominion all'interno del Commonwealth britannico come Unione Sudafricana (Union of South Africa).

Nel XX secolo i testi libreschi, giornalistici, enciclopedici, ecc. non concordano sulla grafia della voce usata per indicare il territorio qui considerato. Sud Africa, Sudafrica e in misura minore Sud-Africa sono utilizzati indifferentemente, come si può dedurre da una rapida indagine condotta con Google Ricerca Libri in rete.

Il Deonomasticon Italicum di Wolfgang Schweickard non presenta la voce Sudafrica, ma s.v. Africa (vol. I, I997) registra sudafricano agg. 'del Sudafrica' (I892) con la variante sud-africano (I96I) e 'abitante, nativo del Sudafrica' (I892) con sud-africano (I99I) e sudafricanizzazione 'l'acquisire caratteristiche e atteggiamenti simili a quelli del Sudafrica, soprattutto per quanto riguarda il problema razziale e il modo di affrontarlo' (I988).

Anche oggi, come del resto valutato già da alcuni lettori che ci scrivono, le varianti sono usate senza distinzione; una banale ricerca con il motore Google, usando nella stringa la parola "stato" per essere certi di isolare i testi italiani, indicherebbe tuttavia un uso della forma univerbata Sudafrica doppio rispetto a Sud Africa.

La nazione delle tre capitali (Pretoria capitale amministrativa, Città del Capo legislativa e Bloemfontein giudiziaria) è anche il Paese delle is lingue ufficiali: due europee e nove locali. In inglese è la Republic of South Africa, in afrikaans Republiek van Suid-Afrika (fino al 1983 - Zuid-Afrika, come in nederlandese, da cui la sigla ZA usata in vari contesti, per esempio nei dominî Internet). Inoltre la voce Africa, resa come Afrika, in un caso Aforika e in un altro Afurika, appare nelle lingue ufficiali Zulu, Xhosa, Tswana, Southern Sotho, Tsonga, Swazi, Venda e Southern Ndebele separata dall'indicatore per 'sud' ed esclusivamente in Northern Sotho unita da un trattino (Afrika-Borwa).

In altre lingue europee il nome dello Stato è documentato nei modi seguenti: francese République d'Afrique du Sud, romeno Republica Africa de Sud, portoghese República da África do Sul, spagnolo República de Sudáfrica, catalano República de Sud-áfrica, tedesco Republik Südafrika, danese Sydafrikanske Republik, svedese Republiken Sydafrika, norvegese Republikken Sør-Afrika, polacco Republika Poludniowei Afryki, basco Hegoafrikako Errepublika, finlandese Etelä-Afrikkalainen tasa valta, 
ungherese Dél-afrikai Köztársaság, croato Južnoafrička Republika, ceco Jihoafrická republika, turco Güney Afrika Cumhuriyeti, ecc. Nel caso in cui il coronimo sia espresso in forma aggettivale, il confronto con le voci corrispondenti per Sudafrica si configura grosso modo con la medesima tipologia.

A parte le prime tre lingue nelle quali il problema non si pone data la costruzione del toponimo, e dove, come in polacco, l'aggettivo per 'meridionale' viene premesso al nome del continente, nelle altre lingue citate ora la voce è univerbata, ora i due elementi sono congiunti da un trattino, più raramente il corrispondente per 'sud' è separato.

Se ne potrebbe concludere che anche in italiano, dove il trattino appare meno in uso, la scriptio continua Sudafrica o (Repubblica) Sudafricana può essere lievemente preferibile, in linea del resto con il nome di un'altra nazione, il Centrafrica o Repubblica Centrafricana (complice qui l'elisione), mentre nelle più generiche indicazioni come Nord Africa, Nord Europa, Nord America, Sud America, Sud Italia, ecc. l'indicatore latitudinale separato è più frequente (ma nulla si oppone all'uso dei corrispondenti univerbati). L'univerbazione è invece quasi la regola per gli aggettivi di riferimento - sudarabico, sudcoreano, sudmongolico, ecc. - ciò che non vale per i relativi coronimi i quali, specie se non molto ricorrenti, sono espressi preferibilmente con la formula preposizionale: Arabia del Sud (o meridionale), Corea del Sud, Mongolia del Sud, ecc.

Una differenziazione legata a territori distinti non pare invece né frequente né utile. Chi volesse specificare il richiamo alla porzione più meridionale dell'Africa, attraverso un coronimo sovranazionale e subcontinentale, eviterebbe qualsiasi rischio rinunciando alla struttura sintattica anglicizzante denotante+denotato ma scrivendo Africa del Sud o Africa meridionale (eventualmente: estrema), in completo disuso per indicare lo Stato. Nel caso dell'aggettivo sarà il contesto a dissipare l'ambiguità dato che, come registra per esempio il GRADIT s.v., sudafricano - datato al I892 - vale "dell'Africa del sud, specie della Repubblica Sudafricana" e "nativo o abitante dell'Africa meridionale o della Repubblica Sudafricana".

\section{Cita come:}

Enzo Caffarelli, Dove vivono i sudafricani?, "Italiano digitale", 2019, XI, 2019/4 (ottobre-dicembre)

DOI: $10.35948 / 2532-9006 / 2020.3260$

Copyright 2019 Accademia della Crusca

Pubblicato con licenza creative commons CC BY-NC-ND 